QUIPURAMAYOC Revista de la Facultad de Ciencias Contables

Vol. 24 N. ${ }^{\circ} 46$ pp. 151-160 (2016) UNMSM, Lima - Perú

ISSN: 1560-9103 (versión impresa) / ISSN: 1609-8196 (versión electrónica)

\title{
RAZONES FINANCIERAS DE LIQUIDEZ EN LA GESTIÓN EMPRESARIAL PARA TOMA DE DECISIONES
}

\author{
FINANCIAL REASONS FOR LIQUIDITY IN BUSINESS MANAGEMENT \\ FOR DECISION MAKING \\ Aiexander Geovanny Herrera Freire* \\ Victor Aib erto Betancourt Gonzaga ** \\ Docentes de ia Universidad Técnica de Machaia - Ecuador \\ Aiex Hum b erto Herrera Freire ${ }^{* * *}$ \\ Docente Universidad Estatal de Mitagro - Ecuador \\ Sara RaquelVega Rodriguez **** \\ Universidad Laica Vicente Rocafuerie de Guayaquil \\ Estefanía Cristina Vivanco Granda *****
}

[Recepción: Abril 2016/ Conformidad: Mayo 2016]

\begin{abstract}
RESUMEN
Los indicadores financieros de liquidez son fundamentales para evaluar la situación y el desempeño económico y financiero de una empresa a corto plazo, es decir, detectar si la empresa tiene suficientes recursos financieros y disponibles para cubrir las obligaciones presentes. El objetivo de este estudio es analizar las razones financieras de liquidez como estrategia en la gestión empresarial para la toma de decisiones. Es un estudio analítico basado en la metodología de (Gitman \& Chad J., 2012) y de revistas científicas las cuales delimitan el criterio de liquidez en una empresa como: "La liquidez representa la agilidad que tiene la empresa para cubrir sus obligaciones financieras a corto plazo", según los autores Linares Mustaros, Farreras Noguer, Ferrer Comalat \& Rabaseda Tares (2012); Marsano Delgado (2013) Morelos Gómez, Fontalvo Herrera \& Hoz Granadillo (2012); Ochoa Ramirez \& Toscano Moctezuma (2012) Vasquez Tejos (2010); entre otras. La importancia de las bases de administración financiera se fundamenta en los ratios para mejorar el análisis financiero y su gestión empresarial. Un correcto análisis de los estados financieros de la organización permite llegar a su actual situación y poder predecir dentro de ciertos límites su desarrollo en el futuro para la toma de decisiones.
\end{abstract}

\section{Palabras Clave:}

Administración Financiera, Gestión empresarial, Toma de decisiones, Indicadores Financieros.

\begin{abstract}
Financial liquidity indicators are fundamental to assess the economic and financial situation and a company's performance in the short term, that is, to detect if the company has sufficient financial resources and is available to cover the present obligations. The objective of this study is to analyze the financial ratios of liquidity as a strategy in business management for decision making. It is an analytical study based on the methodology of Gitman \& Chad (2012) and of scientific journals which delimit the criterion of liquidity in a company as: "The liquidity represents the agility that the company has to cover its financial obligations in the short term", according to Linares Mustaros, Farreras Noguer, Ferrer Comalat \& Rabaseda Tares (2012); Marsano Delgado (2013) Morelos Gómez, Fontalvo Herrera \& Hoz Granadillo (2012); Ochoa Ramirez \& Toscano Moctezuma (2012) Vasquez Tejos (2010), among others. The importance of the financial management foundations is based on the ratios to improving the financial analysis and its business management. A correct analysis of the financial statements of the organization allows us to reach its current situation and enables to predict -within certain limits- its future development for decision making.
\end{abstract}

\section{Keywords:}

Financial Management, Business Management, Decision Making, Financial Indicators.

\footnotetext{
* Doctor en Ciencias Contables y Empresariales Universidad Nacional Mayor de San Marcos Lima-Perú. Mgs en Tributación y Finanzas, Diplomado en tributación en la Universidad de Guayaquil-Ecuador. Email: herrera@utmachala.edu.ec

** Egresado del Doctorado en Ciencias Contables y Empresariales Universidad Nacional Mayor de San Marcos Lima-Perú Magister en Auditoría y Contabilidad de la Universidad Técnica de Machala - Ecuador.Email: vigonzaga@utmachala.edu.ec

*** Mgs en Tributación y Finanzas, Diplomado en tributación Universidad de Guayaquil-Ecuador. Email: betomach_1981@yahoo.es

**** Mgs. en Contabilidad y Auditoría de la Universidad Laica Vicente Rocafuertede Guayaquil -Ecuador. Email: servicioscontableshv@yahoo.es ***** Ingeniera / Contabilidad y Auditoría CPA .Email: stefaniavivanco@hotmail.es
} 
Aiexander Geovanny Herrera Freire / Victor Aib erto Betancourt Gonzaga /

Aiex Hum berto Herrera Freire / Sara Raquel Vega Rodriguez / Estefanía Cristina Vivanco Granda

\section{INTRODUCCIÓN}

Los Indicadores Financieros en el mundo empresarial son imprescindibles para determinar si una empresa puede hacer frente a sus obligaciones a largo plazo, como a corto plazo; mediante un diagnóstico financiero que permite conocer la situación actual de las entidades para realizar una inversión, o para optar por un crédito por parte de una institución financiera; Ochoa Ramirez \& Toscano Moctezuma, (2012) afirma: "Los ratios parten de la idea de la comparación de magnitudes" (p.75), en cualquiera de las decisiones que se adopten en las organizaciones, es importante interpretar cada razón comparándola, utilizando una serie de herramientas para ayudar en el estudio y evaluación consideradas como fundamentales para el análisis de la situación financiera de las mismas.

El papel que desempeña la administración financiera en nuestro país es esencial para el funcionamiento de la economía y por consiguiente para el desarrollo de las empresas en el Ecuador; según Araque J. (2010) nos indica que la aplicación en las empresas de indices financieros (liquidez, endeudamiento, eficiencia y rentabilidad) es del $72.4 \%$ de casos, lo que tiene un efecto beneficioso y por consiguiente incentiva las actividades de inversión o crédito por parte de las instituciones financieras.

Las entidades son sensibles a sufrir alguna variabilidad financiera inesperada, caracterizada por insolvencia y poca liquidez, como fruto de políticas financieras poco efectivas o por insuficiencias en el desempeño estratégico, administrativo, o financiero; consecuentemente toda organización debe conocer su situación económica y financiera para identificar las dificultades existentes y las causas que la ocasionan. Para ello, se debe disponer de herramientas apropiadas que le permitan detectar los errores, a través de este instrumentos predictivos como lo son los ratios financieros, con los que podemos lograr evaluar el desempeño financiero de un ente a lo largo de un ejercicio fiscal especifico, mediante la utilización de fórmulas sobre datos aportados por la contabilidad, Ochoa Ramirez \& Toscano Moctezuma, (2012) afirma: "La técnica de análisis de estados financieros mediante la utilizacion de ratios opera con cociente, es decir, indicadores que son el resultado de la división de una cantidad entre otra (...)” (p.75), y a su vez son convertidos para ser exa- minados e interpretados, con el objeto de alcanzar sus metas y pronósticos proyectados que nos permiten solucionar algunos aspectos concretos en la toma de decisiones para establecer de forma correcta el éxito o fracaso de la organización.

$\mathrm{El}$ análisis financiero a través de ratios, facilita el proceso de toma de decisiones de inversión, financiamiento, planes de acción, lo cual permite identificar los puntos fuertes y débiles de las organizaciones, así como efectuar comparaciones con otras empresas, sean pequeñas o grandes, e imparcialmente de su actividad productiva; ya que contribuye la información necesaria para conocer el proceder operativo de las empresas y su situación económica-financiera, los cuales se fundamentan en los estados financieros bajo las NIIF, según Álvarez Otero \& Rodríguez Enríquez (2011) manifiesta que: "Con la introducción de las NIFF se modifica, potencialmente, la valoración de muchas partidas que integran los estados financieros (...)"(p.12), los cuales son utilizados para calculary examinar los indicadores financieros.

En base a lo que se pronunció anteriormente, se presenta este estudio que tiene como objetivo principal analizar la importancia de las razones financieras de liquidez como estrategia en la gestión empresarial para la toma de decisiones; para ello, es primordial describir la administración financiera y sus principales funciones; identificar los indicadores financieros y sus técnicas en que se fundamenta y analizar los valores calculados de ratios de liquidez como instrumento útil en la gerencia de la empresa estudiada.

La competitividad empresarial según Licona Michel \& Turner Barragán (2014) manifiesta que: "La competitividad es un elemento dinámico, ya que una rentabilidad más alta permite una acumulación más rápida y costos más bajos debido a economías de escala que puede traducirse en una participación cada vez mayor en el mercado, que terminará por hacer desaparecer a las empresas menos competitivas e incapaces de generar rendimientos suficientes" (p.158).

En el ámbito internacional, las empresas nacionales compiten con las compañías transnacionales de diferentes nacionalidades, las cuales adoptan mecanismos que van desde fusiones, alianzas estratégicas, introducción de nuevos productos, incorporación 
de nuevas tecnologías en los productos o servicios, y además el análisis de su liquidez empresarial. Vasquez Tejos (2010) afirma: "El ratio de liquidez nos muestra una aproximación a la variación en el precio que produce por unidad" (p.73). Determinando así la existencia competitiva en el precio del producto o servicio a ofertar una vez utilizado estos parámetros.

Otra ventaja competitiva para las empresas, Según(LINARES MUSTAROS, FARRERAS NOGUER, FERRER COMALAT, \& RABASEDA TARRES, 2012), nos indica que "Permiten analizar la capacidad de absorción del endeudamiento de la empresa en momentos de inestabilidad" (p.40). Este medio es de mucha importancia porque da la oportunidad a la entidad de agilizar sus operaciones comerciales.

\section{FUNDAMENTACIÓN TEÓRICA}

\section{Administración Financiera.-Definición}

En el mundo empresarial actual, se dice que las organizaciones tienen que ser redituables, sustentables y competitivas, y para lograr esto se debe tener una buena administración.

Van Horne \& Wachowicz (2010) afirma: "La administración financiera se ocupa de la adquisición, el financiamiento y la administración de bienes con alguna meta global en mente" (p.2).

Los recursos financieros son de los más importantes dentro de una organización, por ello es necesario usar este recurso de forma adecuada para aprovecharlo de la mejor manera posible en las inversiones que se realizan en un entidad. A continuación describi-

\section{Figura No 01}

Administración Financiera

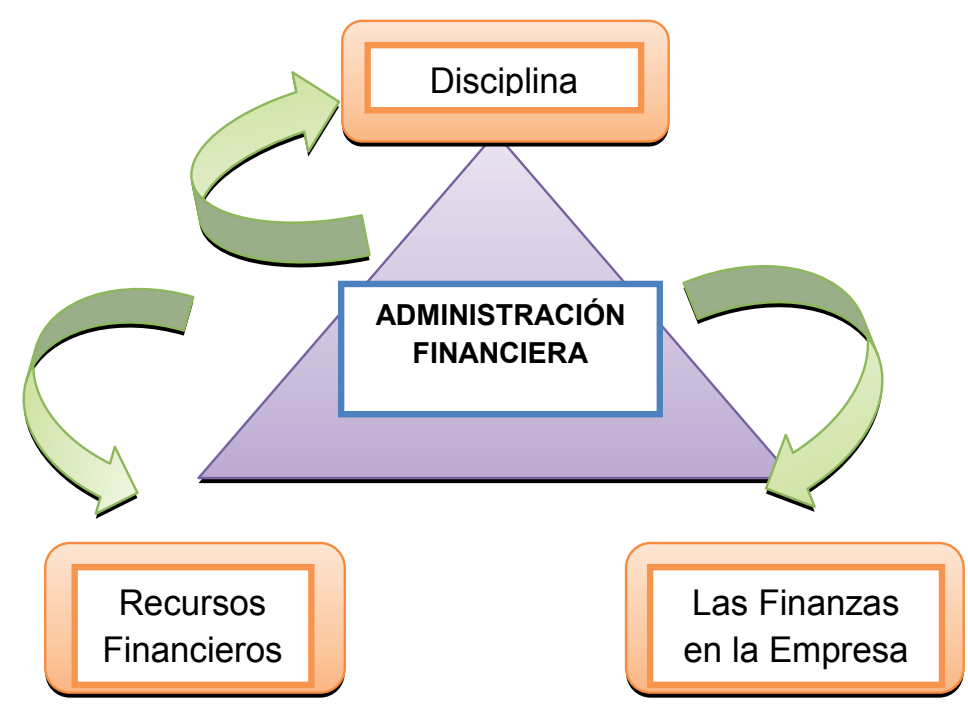

Fuente: Elaboración propia

mos la definición de administración financiera de acuerdo a la Figura $\mathrm{N}^{\circ} 1$, la administración financiera es una disciplina que optimiza los recursos financieros para el logro de los objetivos de la organización con mayor eficiencia y rentabilidad; las cuales son aplicadas a las finanzas en la empresa.

Principales actividades del administrador financiero.

Dentro de la participación continúa en el análisis y la planificación de las finanzas.

Lawrence J. ( 2010) afirma:
"Las principales actividades del administrador financiero son tomar decisiones de inversión y financiamiento” (p.11).

Por lo tanto, las decisiones de inversión determinan tanto la mezcla como el tipo de activos que mantiene la entidad; las decisiones de financiamiento determinan tanto la mezcla como el tipo de financiamiento que usa la empresa. Estas decisiones se estiman convenientes en términos del estado de Situación Financiera, como se muestra en la Figura a continuación: 
Figura No 02:

Actividades Financieras

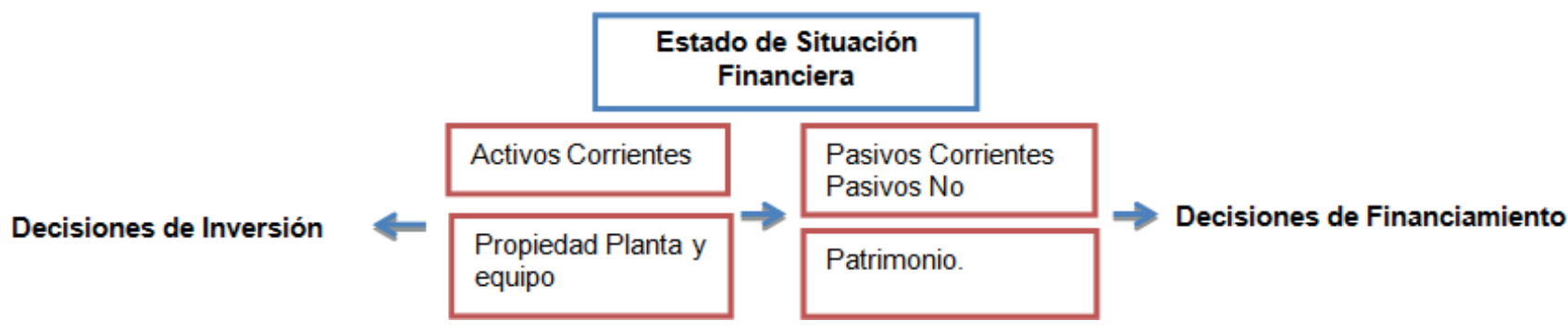

Fuente: Elaboración propia

De acuerdo con la Figura $N^{\circ} 2$, al conferenciar de ratios de liquidez en las actividades financieras, se utilizan los activos y pasivos para determinar las decisiones de inversión y financiamiento que delimitan el futuro económico -financiero de la empresa.

\section{Gestión Empresarial}

Según Sanchez Martorrelli (2013) afirma:

"La gestión empresarial se asocia a las prácticas, organización, sistemas y procedimientos que facilitan el flujo de información para la toma de decisiones, el control, la evaluación y la dirección estratégica del negocio” (p.32).

Además es pertinente considerar la definición de los autores Naranjo, Calderón, \& Álvarez (2011) en donde indica que :

"Gestionar implica ir mas allá de los asuntos operativos y de las contingencias del día a día, es incidir sobre el desarrollo, la innovación y la transformación organizacional" (p.15).

De acuerdo a estos conceptos podemos indicar que la gestión empresarial hace referencia a las medidas estratégicas llevadas a cabo con la finalidad de que la empresa sea viable económicamente, la mismas que tiene en cuenta varios factores, desde lo financiero, pasando por lo productivo hasta lo logístico.

\section{Toma de Decisiones}

Según Robles Roman (2012) manifiesta que:

"Es la técnica de administración financiera que tiene por objeto elegir la mejor alternativa relevante para la solución de problemas en la empresa y para el logro de objetivos preestablecidos, en condiciones de riesgo, conflicto o incertidumbre" (p.15).

En otro sentido Weatley (2011) manifiesta que:

“El proceso de toma de decisiones es un proceso sistemático para identificar y resolver problemas, hacer preguntas y encontrar respuestas (...)" (p.2).

Con lo expuesto anteriormente podemos expresar que toda organización que se encuentre activa y pretenda ser competitiva en el mercado, debe continuamente tomar decisiones gerenciales y de indole financiero tomando como base la información que arrojan los indicadores formulados.

\section{Razones Financieras}

Morelos Gómez, Fontalvo Herrera, \& Hoz Granadillo (2012) manifiesta que:

"Los indicadores financieros son un herramienta para la evaluación financiera de una empresa y para aproximar el valor de esta y sus perspectivas económicas" (p.18).

Las razones financieras también llamados ratios financieros o indicadores financieros, son cifras o razones que facilitan unidades contables y financieras de medida y comparación, a través de las cuales la relación (por división) entre sí de dos datos financieros directos, admiten examinar el estado actual o pasado de una empresa, en función a niveles óptimos delimitados para ella.

Robles Roman (2012) afirma:

“Este método consiste en analizar a los dos estados financieros, es decir, el Estado de Situación Financiera y al Estado de resultados Integral, mediante la combinación entre partidas de un estado financiero o de ambos" (p.33).

Las razones financieras, son comparables con las de competencia y llevan al análisis y reflexión del funcionamiento de las empresas frente a sus rivales.

\section{Categorías de las Razones Financieras}

Por conveniencia las razones financieras se dividen en cuatro categorías básicas: razones de liquidez, actividad, endeudamiento y rentabilidad. 


\section{Razones de Liquidez}

La liquidez de una empresa representa la agilidad que tiene para cumplir con sus obligaciones de corto plazo a medida que estas alcancen su vencimiento. La liquidez se refiere a la solvencia de la situación financiera general de la empresa, es decir, la habilidad con la que puede pagar sus cuentas (Gitman \& Chad J., 2012,p.65).

Bernal, Dominguez L., \& Amat (2012) afirma:

"La liquidez es la capacidad que tiene la empresa para atender sus compromiso de corto plazo" (p.277).

Se refieren no solamente a las finanzas totales de la organización, sino a su destreza para convertir en efectivo determinados activos y pasivos corrientes las dos medidas básicas de liquidez son las siguientes:

\section{Liquidez Corriente}

Este considera la efectiva dimensión de la organización en cualquier instancia del tiempo y es comparable con diferentes empresas de la misma actividad.

Gitman \& Chad J.(2012) afirma:

"La liquidez corriente, mide la capacidad de la empresa

para cumplir con sus obligaciones de corto plazo” (p.65)

, es decir representa la agilidad que tiene una empresa para solventar sus obligaciones en un período menor a un año.

\section{Razón Rápida (Prueba del Ácido)}

Esta prueba es semejante al índice de solvencia, pero dentro del activo corriente no se tiene en cuenta el inventario de productos, ya que este es el activo con menor liquidez.

Gitman \& Chad J.(2012) afirma:

"El nivel de la razón rápida que una empresa debe esforzarse por alcanzar depende en gran medida del sector en la cual opera" (p.67).

Las cuales se expresan en la Tabla $\mathrm{N}^{\circ} 01$ :

Tabla No 01:

Razones de Liquidez

\begin{tabular}{|c|l|l|}
\hline FACTOR & INDICADORES TÉCNICOS & \multicolumn{1}{c|}{ FÓRMULA } \\
\hline LIQUIDEZ & $\begin{array}{l}\text { 1. Liquidez Corriente } \\
\text { 2. Prueba Ácida }\end{array}$ & $\begin{array}{l}\text { Activo Corriente / Pasivo Corriente } \\
\text { Activo Corriente - Inventarios / Pasivo Corriente }\end{array}$ \\
\hline
\end{tabular}

Fuente: Elaboración Propia. www.supercias.gov.ec

\section{Razones de Actividad}

Los Índices de actividad, evalúan la rapidez con la que varias cuentas se transforman en ventas o efectivo, es decir, en entradas o salidas. En cierto sentido, los índices de actividad miden la eficiencia con la que opera una empresa en una variedad de dimensiones, como la administración de inventarios, gastos y cobros, (Gitman \& Chad J., 2012, p.68). Además miden la efectividad con que la empresa esta utilizando los activos empleados.

\section{A continuación su clasificación:}

\section{Rotación de Inventarios}

Gitman \& Chad J. (2012) afirma:

"Mide comunmente la actividad,o liquidez, del inventarios de una empresa” (p.68).

Es decir, mide la liquidez del inventario por medio de su movimiento durante el periodo.

\section{Período Promedio de Cobro}

Gitman \& Chad J. (2012) afirma:

"Es útil para calcular las políticas de crédito y cobro" (p.68).

Se deduce dividiendo el saldo de las cuentas por cobrar entre las ventas diarias promedio;en toda empresa del sector comercial es recomendable calcular el periódo promedio de cobro para logar optimizar de manera eficaz sus políticas de crédito y cobro.

\section{Período Promedio de Pago}

Gitman \& Chad J. ( 2012) afirma:

“Tiempo promedio que se requiere para pagar las cuentas” (p.69).

Es decir, muestra los días que se demora la em- 
Aiexander Geovanny Herrera Freire / Victor Aib erto Betancourt Gonzaga /

Aiex Hum berto Herrera Freire / Sara RaquelVega Rodriguez / Estefanía Cristina Vivanco Granda

presa para pagar sus deudas.

\section{Rotación de los Activos Totales}

Gitman \& Chad J. (2012) afirma:

"Indica la eficacia con la que la entidad utiliza sus activos para generar ventas “(
Por lo tanto, la utilización de sus activos totales (activos corrientes y activos no corrientes) nos muestra la eficiencia que tiene la entidad para asi lograr sus ventas proyectadas.

A continuación el detalle en Tabla $\mathrm{N}^{\circ} 02$ :

Tabla No 02

Razones de Actividad

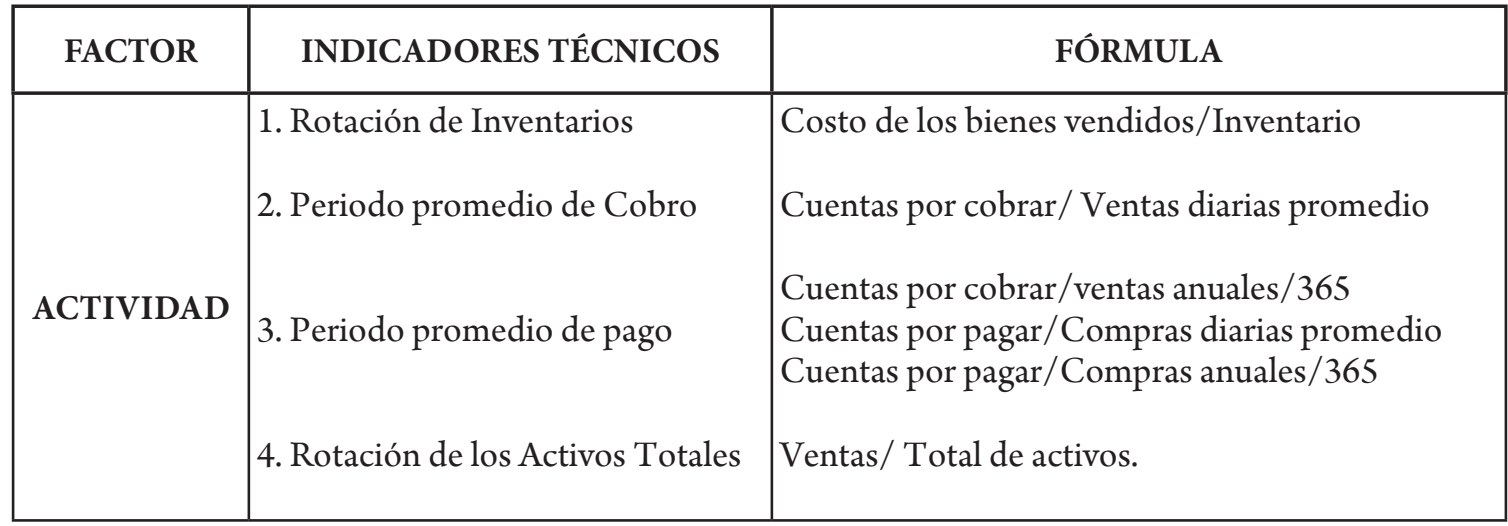

Fuente: Elaboración Propia. www.supercias.gov.ec

\section{Razones de Endeudamiento.}

La posición del endeudamiento de una organización indica el valor del dinero de otras personas que se utiliza para generar utilidades. En general, un analista financiero se interesa más en las obligaciones mayores a un año, por cuanto, estas comprometen a la empresa con un flujo de pagos contractuales a largo plazo (Gitman \&Chad J., 2012, p.70), lo que se detalla mas adelante. (Ver Tabla $\mathrm{N}^{\circ} 03$ )

\section{Razón de cargos de interés fijo.}

Gitman \& Chad J. (2012) manifiesta que: "Mide la Capacidad de la empresa para realizar pago de intereses contractuales. Cuanto más alto es su valor, mayor es la capacidad de la organización para cumplir sus obligaciones de intereses" (p.72).

\section{Índice de cobertura de pagos fijos.}

Gitman \& Chad J.(2012) afirma que el índice de cobertura de pagos fijos: "Nos indica la capacidad de la entidad para cumplir con todas sus deudas de pagos fijos" (p.72). Cuanto más alto es el valor de este índice, se entiende que se esta mejor. Las cuales se expresan a continuación:

Tabla No 03:

Razones de Endeudamiento

\begin{tabular}{|c|c|c|}
\hline FACTOR & INDICADORES TÉCNICOS & FÓRMULA \\
\hline ENDEUDAMIENTO & $\begin{array}{l}\text { 1. Índice de Endeudamiento } \\
\text { 2. Razón de cargos de interés fijo } \\
\text { 3. Índice de cobertura de pagos fijos }\end{array}$ & $\begin{array}{l}\text { Total de pasivos/Total de activos } \\
\text { Utilidad antes de intereses e imp./Intereses } \\
\text { Utilidades antes de intereses e imp. + pagos de } \\
\text { arrendamiento/ Intereses + Pagos de arrenda- } \\
\text { miento }\end{array}$ \\
\hline
\end{tabular}

Fuente: Elaboración Propia. www.supercias.gov.ec

158/ QUIPURAMAYOC | Vol. 24(46) 2016 


\section{Razones de Rentabilidad.}

Estas razones permiten analizar y valorar las ganancias de la entidad con relación a un nivel dado de ventas, de activos o la inversión de los dueños.

Marsano Delgado (2013) manifiesta que:

"Mide la rentabilidad obtenida al cierre del ejercicio económico en función a sus recursos propios"(p.54).

Otros autores Gitman \& Chad J. (2012) afirman:

"Los dueños, los acreedores y la administración prestan mucha atención al incremento de sus utilidades debido al gran valor que el mercado otorga a las ganancias"(p.73).

A continuación la siguiente clasificación:

\section{Margen de Utilidad Bruta}

Gitman \& Chad J. (2012) manifiesta que:

"Mide el porcentaje que queda de cada dólar de ventas después de que la empresa pagó sus bienes. Cuanto mas alto es el margen de utilidad bruta, mejor (ya que es menor el costo relativo de la mercancía vendida)"(p.74).

Por lo tanto, es una medida financiera utilizada para determinar la salud financiera de una empresa.

\section{Margen de Utilidad Operativa}

Mide el porcentaje que queda de cada dólar de ventas después de que se dedujeran todos los costos y gastos, excluyendo los intereses, impuestos y dividendos de acciones preferentes; las utilidades puras ganadas por cada dólar de ventas (Gitman \& Chad J., 2012, p.75). Es decir, es preferible un margen de utilidad operativo alto.

\section{Margen de Utilidad Neta}

Gitman \& Chad J. (2012) afirma:

“ Nos indica el porcentaje que queda de cada dólar de ventas después de que se dedujeron todos los costos y gastos, incluyendo intereses, impuestos y dividendos de acciones preferentes"(p.75).

Es decir, el margen de utilidad neta indica la tasa de utilidad obtenida de las ventas y de otros ingresos.

\section{Ganancias por Acción (GPA)}

Gitman \& Chad J. (2012) manifiesta que:

"Las ganancias por acción (GPA) de la entidad son importantes para los accionistas actuales o futuros y para la administración. La GPA representan el monto en dólares obtenido durante el período para cada acción común en circulación"(p.75).

\section{Rendimiento sobre los Activos Totales (RSA)}

Mide la eficacia integral de la administración para generar utilidades con sus activos disponibles; se denomina también rendimiento sobre la inversión (RSI) (Gitman \& Chad J., 2012, p.76). Es decir, cuanto más alto es el rendimiento sobre activos totales de la entidad mejor. Rendimiento sobre el Patrimonio (RSP)

Gitman \& Chad J. (2012) afirma:

"Mide el rendimiento ganado sobre la inversión de los accionistas comunes en la empresa, cuanto más alto es este rendimiento, más ganan los propietarios” (p.76).

A continuación se expresan en Tabla $\mathrm{N}^{\circ} 04$.

Tabla No 04

Razones de Rentabilidad

\begin{tabular}{|c|l|l|}
\hline FACTOR & INDICADORES TÉCNICOS & \multicolumn{1}{c|}{ FÓRMULA } \\
\hline \multirow{3}{*}{ RENTABILIDAD } & $\begin{array}{l}\text { 1. Margen de utilidad bruta } \\
\text { 2. Margen de utilidad operativa } \\
\text { 3. Margen de utilidad neta }\end{array}$ & Ventas- Costo de los bienes vendidos/ Ventas \\
4. Ganancias por acción & $\begin{array}{l}\text { Utilidad operativa / Ventas } \\
\text { Ganancias disponibles para los accionistas comunes / Ventas } \\
\text { totales (RSA) } \\
\text { Ganancias disponibles para los accionistas comunes } \\
\text { 6. Rendimiento sobre el } \\
\text { Patrimonio ( RSP) }\end{array}$ & $\begin{array}{l}\text { Gunero de acciones comunes en circulación } \\
\text { / Total de activos } \\
\text { Ganancias disponibles para los accionistas comunes / Capital } \\
\text { en acciones comunes }\end{array}$ \\
\hline
\end{tabular}

Fuente: Elaboración Propia. www.supercias.gov.ec 


\section{Caso Práctico de Razones Financieras de Liquidez}

La empresa Freemont Corporation posee un efectivo de $\$ 1500.00$, inversiones temporales de $\$ 2500.00$, cuentas por cobrar de $\$ 15000.00$, inventarios $\$ 33000.00$, activo fijo de $\$ 35000.00$, cuentas por pagar $\$ 12500.00$, títulos por pagar $\$ 12500.00$, deuda a largo plazo $\$ 22000.00$, acciones comunes $\$ 5000.00$, capital $\$ 18000.00$, utilidades retenidas $\$ 17000.00$.

Calcule las siguientes razones financieras de liquidez:
a. Razón Circulante
b. Prueba Ácida
Resultados

El Estado de Situación Financiera contiene información sobre los activos, pasivos y capital contable de Freemont Corporation. Las cifras proporcionan una visibilidad de la realidad financiera de la empresa al 31 de Diciembre del año 2014. Los activos están registrados al precio que la empresa pagó por ellos, es decir, al costo histórico. Los pasivos son los valores que la entidad debe a sus acreedores y el capital contable, también llamados valor neto o capital de propietarios, además es la diferencia entre activos y pasivos totales. A continuación se utilizan las razones financieras de liquidez para examinar la capacidad que tiene Freemont Corporation para cubrir sus obligaciones a corto plazo por vencer, las cuales se determinan en la Tabla $\mathrm{N}^{\circ} 05$ :

Tabla No 05

Razones de Liquidez de Freemont Corporation

\begin{tabular}{|c|c|c|c|c|}
\hline FACTOR & $\begin{array}{c}\text { INDICADORES } \\
\text { TÉCNICOS } \\
\end{array}$ & FÓRMULA & CALCULO & RESULTADO \\
\hline LIQUIDEZ & $\begin{array}{l}\text { 1.Liquidez Corriente } \\
\text { 2.Prueba Ácida }\end{array}$ & $\begin{array}{c}\text { Activo Corriente / } \\
\text { Pasivo Corriente } \\
\text { Activo Corriente - Inventarios / } \\
\text { Pasivo Corriente }\end{array}$ & $\begin{array}{c}52.000 / 25.000 \\
52.000-33.000 / 25.000\end{array}$ & $\begin{array}{l}2.08 \\
0.76\end{array}$ \\
\hline
\end{tabular}

Fuente: Elaboración Propia. Estado de Situación Financiera de Freemont Corporation.

\section{Figura No 03}

Razones Financieras de Liquidez de Freemont Corporation

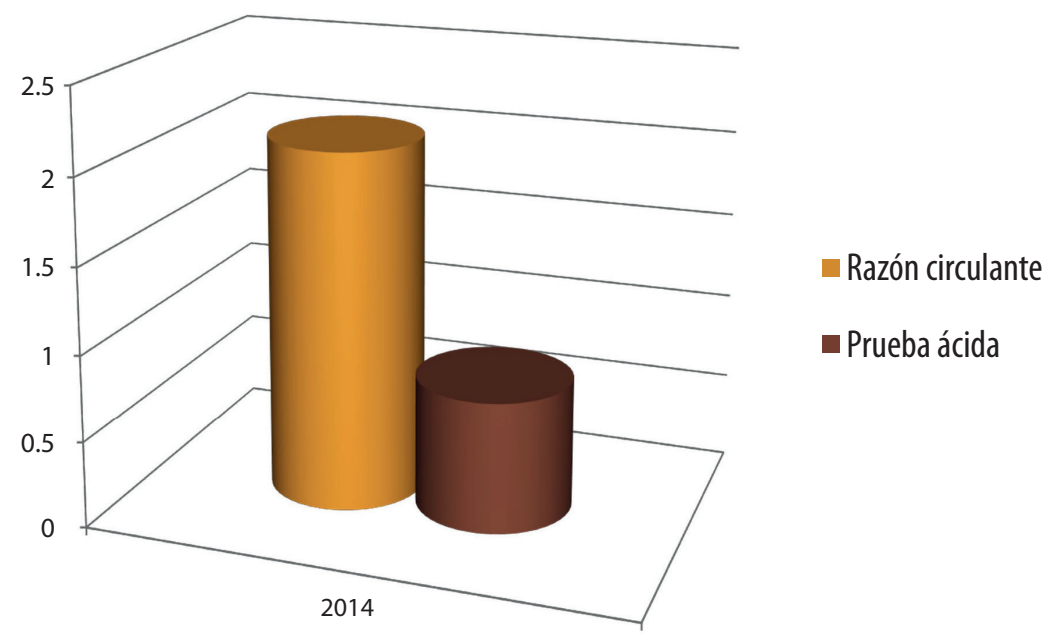

Fuente: Elaboración propia. 


\section{Interpretación Razón Corriente:}

Este índice mide el resultado de la empresa para cubrir oportunamente sus obligaciones a corto plazo, en términos generales un índice de solvencia de 1 se considera aceptable en una empresa comercial (Gitman \& Chad J., 2012, p.65). Como podemos observar en la Figura $N^{\circ} 3$, la razón circulante de Freemont Corportation para el final del año 2014 es de 2.08, por lo tanto el activo circulante cubre dos veces su pasivo circulante. La razón se interpreta en el sentido de que por cada dólar de deuda la empresa cuenta con 2.08 para cancelar su obligación corriente; es decir, debe estar en posibilidad de convertir cada dólar del activo circulante en al menos 0.48 dólares de efectivo $(\$ 1.00 / 2.08=0.48)$; de tal manera que Freemont Corporation posee una solvencia favorable, por ende cuenta con la suficiente liquidez para cumplir con sus obligaciones.

\section{Razón de Prueba Ácida}

Este ratio muestra una medida de liquidez más precisa que la anterior, ya que excluye las existencias para cubrir sus obligaciones corrientes, por lo tanto no debe ser inferior a 1 (Gitman \& Chad J., 2012, p.66). De acuerdo a la Figura $\mathrm{N}^{\circ} 3$ los resultados obtenido en la prueba ácida de la empresa es de 0.76 en el año 2014, lo cual quiere decir que por cada dólar que Freemont Corporation debe a corto plazo dispone de $0.76 \mathrm{ctvs}$; sin tener que contar con los Inventarios los cuales representan el 63\% de sus respectivos activos corrientes y no son considerados para al cálculo debido a que son los menos líquidos, se supone que el resultado ideal sería la relación 1:1, un dólar que se debe y un dólar que se tiene para pagar, esto garantizaría el pago de la deuda a corto plazo y llenaría de confianza a cualquier acreedor; resultados que son poco satisfactorios para solventar las obligaciones financieras vigentes, por lo tanto los representantes de la empresa deberán realizar lo necesario para mejorar sus rentabilidad (ingresos) y controlar sus costos y gastos.; y así poder afrontar sus obligaciones menores a 360 días.

\section{CONCLUSIONES}

1. La administración financiera como fundamento en la aplicación de los índices, ratios e interpretación financiera logra una gestión empresarial positiva.

2. Se ha detectado el desconocimiento que existe sobre la aplicación y la gestión financiera por parte de socios, accionistas, gerentes administrativos o financierosy de los administradores de las empresas para la toma de decisiones.

3. El problema del sector empresarial al calificar su posición financiera a corto plazo, se determina en la medida que presenta la rentabilidad para cubrir las obligaciones presentes; de manera que, un correcto análisis de los estados financieros de la organización va a permitir identificar la actual situación económica y predecir su desarrollo futuro, a través de decisiones correctas enfocadas en datos financieros confiables.

4. La empresa Freemont Corporation obtuvo como efecto una liquidez corriente muy buena por cuanto, de al resultado obtenido, sus recursos disponibles pueden solventar las obligaciones presentes, a diferencia de la prueba ácida, el hecho de que esta última razón este por debajo del promedio, no significa que la empresa debe considerar el cese de su actividad económica para así satisfacer la demanda de los acreedores de corto plazo; al igual que una razón muy alta significa más tranquilidad para los acreedores, pero para la gerencia de la entidad representa muchos recursos inmovilizados; ya que estas razones proporcionan un parámetro para medir su liquidez; y es el analista financiero quien debe ir al fondo de la razón financiera para descubrir porque difiere del promedio y determinar si existe un problema grave.

Se pretende que el presente trabajo haya mostrado la importancia de este tipo de análisis, necesario en cualquier estudio empírico que se debe usar adecuadamente, y que consecuentemente en los futuros estudios económico financieros sean incluidos nuevas razones financieras como las propuestas, complementando así la información que ofrecen los estudios tradicionales en áreas de una mayor determinación de la solvencia empresarial. 


\section{REFERENCIAS BIBLIOGRÁFICAS}

1. ÁLVAREZ, S. \& RODRÍGUEZ, E. (2011). La valoración Bursátil en el marco de las NIIF. Análisis Financiero, 89.

2. ARAQUE, W. (2010). Prácticas de la gerencia financiera en la empresa ecuatoriana. Quito: $\mathrm{Ob}$ servatorio PYME.

3. BERNAL, D. ; DOMINGUEZ, L. \& AMAT, O. (2012). Anuarios de ratios financieros sectoriales en México para análisis comparativo empresarial. Ra Ximhai, 286.

4. GITMAN, L. \& CHAD J, Z. (2012). Principios de Administración Financiera (Décimosegunda Edición ed.). México: Pearson Educación.

5. LAWRENCE, G. (2010). Administración Financiera. Mexico: Pearson Eduación.

6. LICONA, Á. \& TURNER, E. (2014). Competitividad Sistemática y pilares de la competitividad de Corea del Sur. Revista Análisis Económico, 175.

7. LINARES MUSTAROS, S., FARRERAS NOGUER, M. A., FERRER COMALAT, J. C., \& RABASEDA TARRES, J. (2012). Una Nueva Ratio Sectorial. La ratio de retorno liquido. Cuadernos del CIMBAGE N $15,72$.

8. MARSANO, J. (2013). El análisis financiero y su importancia en la gestión de empresas turísticas. Revista Económica Perú, 63.

9. MORElOS, J. ; FONTAlVO, T. \& HOZ, E.
(2012). Análisis de los Indicadores Financieros en las Sociedad Portuarias de Colombia. Revista Entramado, 26.

10.NARANJO, J.; CALDERÓN, G. \& ÁLVAREZ, C.(2011). Gestión empresarial en Colombia: un aporte desde la administración. Universidad Nacional de Colombia, 125.

11.OCHOA, S. \& TOSCANO, J. (2012). Revision crítica de la literatura sobre el análisis financiero de las empresas. Revista de Ciencias Sociales y Humanidades, 99.

12.ROBLES, C. (2012). Fundamentos de Administracion Financiera. México: Red Tercer Milenio S.C.

13.SANCHEZ, J.(2013). Indicadores de Gestión Empresarial. Estados Unidos de América: Palibrio LLC.

14.VAN, J. \& WACHOWICZ, J. (2010). Fundamentos de Administracion Financiera. México: Pearson Educación.

15.VASQUEZ, F. (2010). Riesgo de Liquidez en el mercado bursátil chileno. Análisis Financiero, 81.

16.WEATLEY, G. (2011). Toma de Decisiones. Lifelong Learning Programme, 12.

\section{REFERENCIAS ELECTRÓNICAS}

1. www.supercias.gob.ec 